\title{
Seguimiento analítico, radiológico y oximétrico de los pacientes con COVID-19 como guía para su manejo terapéutico
}

\author{
Analytical, radiological and oxymetric follow-up \\ of patients with COVID 19 as a guide for their \\ therapeutic management
}

\author{
Luis López-Yepes, MSc ${ }^{1}$; Ana Urrutia Brán, MSc ${ }^{2}$
}

\section{Resumen}

Introducción: la COVID-19 es una enfermedad infecciosa que puede manifestarse desde una infección banal de la vía aérea superior (60 a $70 \%$ de los casos) hasta un síndrome de distrés respiratorio leve, moderado o grave, así como un daño orgánico multisistémico (40\% de los casos, 88 $\%$ de los cuales tienen uno o más factores de riesgo). El desarrollo de la llamada "tormenta de citoquinas" ocurre en los días 5 a 7 de la enfermedad y depende a su vez de la presencia o no de factores de riesgo. Por tanto, es posible crear un modelo predictivo del desarrollo de dicha tormenta de citoquinas y la probabilidad de progresión a formas pulmonares graves, para administrar el tratamiento antiviral y antiinflamatorio enérgicamente, en los momentos de la enfermedad en que son más efectivos.

Problema: el desarrollo del Síndrome de Distrés Respiratorio Agudo (SDRA) por el nuevo coronavirus SARS-CoV-2 es de difícil manejo y tiene una elevada morbimortalidad.

Objetivo: plantear un modelo predictivo de riesgo de desarrollo de SDRA por el nuevo coronavirus SARS-CoV-2 desde sus fases iniciales, según factores de riesgo y datos analíticos, clínicos y radiológicos, para administrar los medicamentos antivirales $\mathrm{y} / \mathrm{o}$ antiinflamatorios en momentos más oportunos, evitando así el paso a las fases avanzadas de la enfermedad, como el SDRA o el fracaso multiorgánico, en vez de tratar al paciente una vez se ha instalado el daño.

Material y métodos: cohorte de 796 pacientes, multicéntrica, vistos desde abril de 2019 al $1^{\circ}$ de abril de 2021, en un hospital terciario privado de Guatemala, un hospital terciario público y pacientes vistos en la clínica privada.

Resultados y discusión: del total de pacientes, el $56 \%$ tuvo un curso clínico leve, $29 \%$ tuvo una tormenta de citoquinas leve, con el desarrollo
${ }^{1}$ Neumólogo del Hospital San Vicente, Hospital El Pilar y clínica privada. Ciudad de Guatemala, Guatemala. dr.lopezyepes@yahoo.com

${ }^{2}$ Médico nutrióloga, MSc en epidemiologia y estadística, clínica privada.

Ciudad de Guatemala, Guatemala.

Recibido: $26 / 02 / 2021$

Aceptado: 30/11/2021 
de una neumonía leve y el $15 \%$ una tormenta de citoquinas moderada o grave, con el desarrollo de SDRA leve o moderado. Hubo una mortalidad del $0.36 \%$. Los pacientes con curso clínico leve no tuvieron factores de riesgo de mala progresión y fueron tratados de forma sintomática y ambulatoria. Los pacientes con factores de riesgo de mala progresión fueron tratados con remdesivir desde el diagnóstico. Los que desarrollaron una tormenta de citoquinas leve fueron tratados con glucocorticoides y anticoagulación en dosis bajas a moderadas. Los pacientes con tormenta de citoquinas moderada o grave, fueron tratados con bolos de glucocorticoides en dosis moderadas o altas, anticoagulación, con o sin tocilizomab y remdesivir, dependiendo de los valores de interleucina 6 y de la carga viral detectada. Ninguno tuvo efectos secundarios de gravedad con el tratamiento.

Conclusiones: iniciar un tratamiento precoz y oportuno en pacientes con factores de riesgo y/o que desarrollen una tormenta de citoquinas puede evitar el desarrollo del distrés respiratorio y reducir su gravedad en pacientes con COVID-19, reduciendo la necesidad de oxigenoterapia a corto y largo plazo, la estancia hospitalaria, el ingreso a unidad de cuidados intensivos (UCI), la necesidad de ventilación mecánica, la mortalidad y secuelas a largo plazo.

Palabras clave: COVID-19; tratamiento; glucocorticoides; tocilizumab; remdesivir.

\begin{abstract}
Introduction: COVID-19 is an infectious disease that can manifest itself from a banal infection of the upper airway (60 to $70 \%$ of cases) to a mild, moderate or severe respiratory distress syndrome, as well as multisystem organ damage ( $40 \%$ of cases, $88 \%$ of which have one or more risk factors). The development of the so-called "cytokine storm" occurs on days 5 to 7 of the disease, and depends in turn on the presence or not of risk factors. Therefore, it is possible to create a predictive model of the development of such a cytokine storm and the likelihood of progression to severe pulmonary forms, to administer antiviral and anti-inflammatory treatment vigorously, at the times of the disease when they are most effective.
\end{abstract}

Problem: The development of ARDS by the new coronavirus SARS COV-2 is difficult to manage and has a high morbidity / mortality.

Objective: To propose a predictive model of risk of ARDS development by the new SARS COV-2 coronavirus from its initial phases, according to risk factors and analytical, clinical and radiological data, to administer antiviral and / or anti-inflammatory drugs at more opportune times, thus avoiding the passage to the advanced stages of the disease, such as ARDS or multisystem organ damage, instead of treating the patient once the damage has been installed.

Material and methods: Cohort of 796 patients, multicenter, seen from April 2019 to April 1, 2021, in a private tertiary hospital in the country, a public tertiary hospital and patients seen in the private clinic.

Results and discussion: Of the total patients, 56\% had a mild clinical course, $29 \%$ had a mild cytokine storm, with the development of mild pneumonia, and 15\% a moderate or severe cytokine storm, with the development of mild or moderate ARDS. There was a mortality of $0.36 \%$. Patients with mild clinical course had no risk factors for poor progression and were treated symptomatically and on an outpatient basis. Patients with risk factors for poor progression were treated with remdesivir from diagnosis. Those who developed a mild cytokine storm were treated with glucocorticoids and anticoagulation at low to moderate doses. Patients with moderate or severe cytokine storm were treated with glucocorticoid bolos at moderate or high doses, anticoagulation, with or without tocilizomab and remdesivir, depending on interleukin 6 values and detected viral load. None had serious side effects with treatment.

Conclusions: Initiating early and timely treatment in patients with risk factors and/or who develop a cytokine storm can prevent the development of respiratory distress and reduce its severity in patients with COVID-19, reducing the need for oxygen therapy in the short and long term, hospital stay, admission to the ICU, the need for mechanical ventilation and mortality and long-term sequelae.

Keywords: Covid 19, treatment, glucocorticoids, tocilizumab, remdesivir. 


\section{Introducción}

La COVID-19 es una enfermedad infecciosa que puede manifestarse de varias formas; desde una infección banal de la vía aérea superior hasta un síndrome de distrés respiratorio-SDRA leve, moderado o grave, así como un daño orgánico multisistémico. Ha alcanzado una mortalidad global cercana al $6 \%$ que varía según la región geográfica, llegando a ser de 10 a $12 \%$ en algunos países europeos y del $2 \%$ en algunos países de América y África. De los pacientes que desarrollan neumonías moderadas y graves, entre $10 \%$ a $20 \%$ puede quedar con secuelas pulmonares permanentes, como el desarrollo de enfermedad pulmonar intersticial y la necesidad de oxigenoterapia complementaria crónica.

Puede dividirse en tres momentos o fases: el primero, con síntomas de vía aérea superior, leves, entre los días 1 a 6 de síntomas. El segundo (días 5 a 12) con un desarrollo o no de una tormenta de citoquinas. El tercero (día 6 en adelante) con la instauración o no de un síndrome de distrés respiratorio y/o fracaso multiorgánico variable en su gravedad. El desarrollo de la llamada "tormenta de citoquinas" se manifiesta como una elevación de la interleucina 6 (IL-6), deshidrogenasa láctica (DHL), ferritina, proteína $\mathrm{C}$ reactiva (PCR), linfopenia, reducción de 1 a $2 \%$ en la saturación de oxígeno (SpO2) basal, taquicardia o cambios incipientes en los Rayos X de tórax. Ocurre en los días 5 a 7 de la enfermedad y depende a su vez de la presencia o no de factores de riesgo. Entre estos los más importantes son la obesidad, la diabetes mellitus mal controlada, el síndrome de apnea obstructiva del sueño mal controlado (SAOS), la hipertensión arterial (principalmente asociada a obesidad), ser de sexo masculino y tener una edad por encima de los 60 años.

Por tanto, es posible crear un modelo predictivo del desarrollo de dicha tormenta de citoquinas y la probabilidad de progresión a formas pulmonares graves, para administrar el tratamiento antiviral y antiinflamatorio oportunamente, en los momentos de la enfermedad en que son más efectivos (1) los antivirales como el remdesivir (2) en los primeros días de la enfermedad (cuando hay una mayor carga viral) en los pacientes con factores de riesgo; los antiinflamatorios esteroideos, tocilizumab $(1,3) \mathrm{u}$ otros inmunosupresores, en el momento en que se detecte el inicio de una tormenta de citoquinas (según la analítica, datos clínicos o radiológicos) administrando uno $\mathrm{y} / \mathrm{u}$ otro, con dosis ajustadas a la gravedad de la inflamación registrada de dicha tormenta (Ver Tabla 1). Esto suele ocurrir en torno al quinto o sexto día de la enfermedad, principalmente en los pacientes con factores de riesgo de mala progresión.

Los datos analíticos, clínicos o radiológicos que describen el inicio de una tormenta de citoquinas pueden advertir, según la magnitud de sus alteraciones y principalmente en pacientes con los factores de riesgo mencionados, el desarrollo de un SDRA en las 48 a 72 horas posteriores. $\mathrm{Al}$ administrar los fármacos inmunosupresores y corticoideos en esta etapa, se puede evitar el paso a SDRA moderado o grave, así como el desarrollo de fracaso multiorgánico, al detener la tormenta de citoquinas desde sus fases iniciales, en vez de esperar a que el daño ya se haya instalado, para administrarlos. Esto promueve que la evolución de la enfermedad curse con una inflamación de menor magnitud, con menor daño pulmonar u otras secuelas crónicas irreversibles.

Tocilizumab: es un anticuerpo monoclonal humanizado capaz de interferir con el sitio de unión a membrana y el receptor de IL-6, bloqueando así el ensamblaje del complejo activado con la proteína transmembrana.

El tocilizumab también es capaz de bloquear la transeñalización de IL-6, que está fuertemente relacionada con los efectos proinflamatorios. Tiene un perfil farmacocinético no lineal, con una curva dosis-respuesta que se estabiliza a una dosis aproximada de $800 \mathrm{mg}$. Según se reportó en el estudio de Toniati et al., dado el papel que tiene la interleuciona 6 en el estado proinflamatorio, luego de la administración de tocilizumab los reactantes de fase aguda disminuyeron y los pacientes alcanzaron gradualmente una condición estable (1).

Remdesivir: medicamento antiviral que pertenece al grupo de los análogos de nucleótidos y compite con el sustrato adenosina trifosfato (ATP) natural para la incorporación en las cadenas de ácido desoxirribonucleico (ADN) nacientes por la ADN polimerasa dependiente de ácido ribonucleico (ARN) del SARS-CoV-2, lo que resulta en la terminación retardada de la cadena durante la replicación del ADN viral (4). Como se menciona en el estudio de Sreekanth Reddy et al., recientemente se ha descubierto que el remdesivir inhibe la replicación del SARS-CoV-2 en células pulmonares humanas y en células epiteliales 
primarias de las vías respiratorias (5).

Metilprednisolona: glucocorticoide que interactúa con receptores citoplasmáticos intracelulares específicos que penetran en el núcleo de la célula, donde interactúa con secuencias específicas de ADN, que estimulan o reprimen la transcripción génica de ARNm específicos que codifican la síntesis de determinadas proteínas en órganos diana, alcanzando a dosis farmacológicas acciones antiinflamatorias e inmunosupresoras (6).

Enoxaparina: heparina de bajo peso molecular (HBP) que inhibe la coagulación potenciando el efecto inhibitorio de la antitrombina III sobre los factores IIa y Xa, con una mayor actividad en este último (7).

Rivaroxaban/ Edoxaban: son inhibidores directos del factor Xa altamente selectivos. La inhibición del factor Xa interrumpe las vías intrínsecas y extrínsecas de la cascada de la coagulación, inhibiendo así la formación de trombina, como la formación de los trombos (8).

\section{Materiales y métodos}

Se trata de un estudio descriptivo, retrospectivo, tipo de serie de casos de una cohorte de 796 pacientes, multicéntrica, vistos desde abril de 2019 hasta el $1^{\circ}$ de abril de 2021, en un hospital terciario privado del país, un hospital terciario público, en la clínica privada y en seguimiento por telemedicina, en los casos leves. Se procuró realizar un diagnóstico precoz de COVID-19 por PCR nasal. En los primeros días se realizó una analítica en ayunas, examen físico e historial clínico para detectar los factores de riesgo de mala progresión de la COVID-19 (diabetes mellitus mal controlada, hipertensión arterial, obesidad, síndrome de apnea obstructiva del sueño, edad mayor de 60 años y sexo masculino). Los días de enfermedad se contaron según el primer día de aparición de síntomas. A los pacientes con uno o más de los seis principales factores de riesgo de mala progresión de la enfermedad, se les administró desde el inicio remdesivir, en dosis inicial de 200 $\mathrm{mg}$, para continuar a $100 \mathrm{mg}$ cada 24 horas por 5 a 10 días, dependiendo de la evolución clínica en los días siguientes. Los pacientes recibieron seguimiento mediante historia clínica, medición diaria de la SpO2, frecuencia cardíaca, presión arterial y glucometría (en los que tuviesen intolerancia a la glucosa o diabetes mellitus). Entre los días 5 a 7 se realizó una analítica de control y una radiografía de tórax, para vigilar la aparición de una tormenta de citoquinas. Los datos analíticos que se consideraron predictores del desarrollo de una tormenta de citoquinas fueron los siguientes (Ver Tablas 1 y 2).

Si se encontraban datos de probable progresión hacia fases de SDRA iniciales, como una caída de la SpO2 basal en un 1 a $2 \%$, taquicardia, nuevas lesiones pulmonares en la radiografía de tórax o elevación de los reactantes de inflamación como interleucina 6, ferritina, PCR o una caída de los linfocitos, se realizaba un ingreso hospitalario del paciente para continuar la monitorización, y se iniciaba tratamiento

Tabla 1. Datos analíticos predictores de tormenta de citoquinas .

\begin{tabular}{|lll|}
\hline \multicolumn{1}{|c}{ Dato Analítico } & \multicolumn{1}{c|}{$\begin{array}{c}\text { Tormenta Citoquinas } \\
\text { Leve }\end{array}$} & \multicolumn{1}{c|}{$\begin{array}{c}\text { Tormenta Citoquinas Moderada a } \\
\text { grave }\end{array}$} \\
PCR & $20-50 \mathrm{mg} / 1$ & $>50 \mathrm{mg} / \mathrm{l}$ \\
$\mathrm{IL6}$ & $7-30 \mathrm{pg} / \mathrm{mL}$ & $>50 \mathrm{pg} / \mathrm{mL}$ \\
DHL & $200-300 \mathrm{U} / \mathrm{L}$ & $>500 \mathrm{U} / \mathrm{L}$ \\
Linfopenia & $1000-1500 \mathrm{células} / \mu \mathrm{l}$ & Menos de $1000 \mathrm{células} / \mu \mathrm{l}$ \\
Dímeros D & $500-1000 \mathrm{ug} / \mathrm{mL}$ & Más de $1000 \mathrm{ug} / \mathrm{mL}$ \\
Ferritina & $200-800 \mathrm{ng} / \mathrm{mL}$ & $>800 \mathrm{ng} / \mathrm{mL}$ \\
\hline
\end{tabular}

PCR: proteína C reactiva, IL-6: interleucina 6, DHL: deshidrogenasa láctica 
Tabla 2. Datos analíticos predictores de tormenta de citoquinas

\begin{tabular}{|lll|}
\hline $\begin{array}{c}\text { Dato clínico o radiológico } \\
\text { (Correlacionarlo con el grado de } \\
\text { elevación de reactantes inflamatorios) }\end{array}$ & Tormenta Citoquinas Leve & $\begin{array}{c}\text { Tormenta Citoquinas } \\
\text { Moderada a grave }\end{array}$ \\
SpO2\% & Caída de 1 a 2\% respecto a la basal & $\begin{array}{l}\text { Caída de más de un } 5 \% \\
\text { respecto a la basal }\end{array}$ \\
Frecuencia cardíaca & $\begin{array}{l}\text { Taquicardia de más de } 100 \text { latidos por } \\
\text { minuto en reposo }\end{array}$ & $\begin{array}{l}\text { Taquicardia de más de } 130 \\
\text { latidos por minuto en reposo }\end{array}$ \\
& $\begin{array}{l}\text { Patrón intersticial de reciente aparición } \\
\text { que ocupa menos de un 20\% de cada } \\
\text { pulmón }\end{array}$ & $\begin{array}{l}\text { Patrón intersticial de reciente } \\
\text { aparición que ocupa más del }\end{array}$ \\
Rayos X de tórax & 20\% de cada pulmón \\
\hline
\end{tabular}

con tocilizumab (si había elevación de la interleucina 6) y bolos de metilprednisolona, en dosis de 80 $\mathrm{mg} / \mathrm{kg} /$ día y 250 a $1000 \mathrm{mg}$ cada 24 horas, por una a 3 dosis, dependiendo de la magnitud de la elevación de reactantes de fase aguda y del deterioro clínico respiratorio del paciente (4). Asimismo, se administró una heparina de bajo peso molecular en dosis profilácticas o terapéuticas, calculada según el riesgo trombótico del paciente según parámetros clínicos y analíticos, como dímero D. Cabe destacar que según varios estudios publicados principalmente la experiencia en Cremona, realizado por Sophie Testa, et al., los niveles de los anticoagulantes directos aumentan significativamente si se utilizan en combinación con antivirales tipo remdesivir, por lo que cuando se ha utilizado este último, la anticoagulación recibida por el paciente ha sido una heparina de bajo peso molecular. Solamente en los pacientes sin factores de riesgo y con COVID-19 leve que desarrollaron tormenta de citoquinas leve se utilizaron anticoagulantes directos (9). A todos se les administró un tratamiento gastroprotector con un inhibidor de la bomba de protones ajustando la dosis al riesgo de gastrolesión individual, así como antibioterapia profiláctica y tratamiento con insulina basal y de rescate, según los valores del glucómetro en el seguimiento. Los datos se analizaron en el programa estadístico SPSS versión 22.0.

\section{Resultados}

De la cohorte de 796 pacientes, el $56 \%$ tuvo un curso clínico leve con afectación exclusiva de la vía aérea superior, $35 \%$ presentó neumonía leve sin distrés respiratorio, con elevación ligera de los reactantes inflamatorios y trombóticos y un $8 \%$ tuvo un distrés respiratorio leve a moderado, con elevación moderada a grave de dichos reactantes.

Sin factores de riesgo y sin desarrollo de tormenta de citoquinas: los pacientes con curso clínico leve, sin neumonía ni elevación de citoquinas, fueron tratados únicamente, con hidratación, acetaminofén y complementos de vitamina C, D y zinc. Todos tuvieron una recuperación completa, sin secuelas.

Sin factores de riesgo clínicos y tormenta de citoquinas leve: los pacientes que en el seguimiento al día 5 o 6 tuvieron caídas en la SpO2 > 1 a $2 \%$ de su basal, elevación de la frecuencia cardíaca encima de $90 \mathrm{lpm}$, o alteraciones analíticas sugestivas del desarrollo incipiente de una tormenta leve de citoquinas, fueron tratados de forma rápida y oportuna con metilprednisolona o prednisona a dosis de 1 a $2 \mathrm{mg} / \mathrm{kg}$ al día y anticoagulación con heparinas de bajo peso molecular o anticoagulantes directos. Todos tuvieron una respuesta completa al tratamiento en menos de cinco días, sin necesitar oxigenoterapia complementaria al alta.

Sin factores de riesgo clínicos y tormenta moderada o grave de citoquinas: los pacientes que tuvieron durante su seguimiento caídas en la SpO2 $>5 \%$ de su basal, elevación de la frecuencia cardíaca encima de $100 \mathrm{lpm}$, o alteraciones analíticas sugestivas del desarrollo incipiente de una tormenta moderada o grave de citoquinas, fueron tratados con 
metilprednisolona a dosis de 250 a $1000 \mathrm{mg}$ cada 24 horas por 3 a 5 días, remdesivir $200 \mathrm{mg}$ el primer día y $100 \mathrm{mg}$ al día por 5 a 10 días, tocilizumab $8 \mathrm{mg} / \mathrm{kg} /$ día, repitiendo su dosis al día siguiente si persistía la inflamación y anticoagulación con HBPM. De ellos, solamente el $4 \%$ requirió oxigenoterapia domiciliaria durante tres meses, ya retirada actualmente. Ninguno necesitó soporte ventilatorio no invasivo o invasivo, oxigenoterapia en alto flujo, ni hubo fallecidos.

Con uno o más de los factores de riesgo clínicos y tormenta de citoquinas leve: se inició remdesivir 200 mg el primer día, desde el momento del diagnóstico y se continuó con $100 \mathrm{mg}$ al día por 5 a 10 días. Los pacientes que tuvieron durante su seguimiento caídas en la SpO2 > 1 a $2 \%$ de su basal, elevación de la frecuencia cardíaca encima de $100 \mathrm{lpm}$, o alteraciones analíticas sugestivas del desarrollo incipiente de una tormenta leve de citoquinas, fueron tratados con metilprednisolona o prednisona a dosis de 1 a $2 \mathrm{mg} /$ $\mathrm{kg}$ al día y anticoagulación con heparinas de bajo peso molecular (HBPM), según los factores de riesgo protrombóticos del paciente y sus niveles de dímero D. Ninguno necesitó oxigenoterapia de alto flujo, soporte ventilatorio invasivo, ni hubo fallecidos.

Con uno o más de los factores de riesgo clínicos y tormenta moderada o grave de citoquinas: se inició remdesivir $200 \mathrm{mg}$ el primer día, desde el momento del diagnóstico y se continuó con $100 \mathrm{mg}$ al día por 5 a 10 días. Los pacientes que tuvieron durante su seguimiento caídas en la SpO2 $>5 \%$ de su basal, elevación de la frecuencia cardíaca encima de 120 lpm, o alteraciones analíticas sugestivas del desarrollo incipiente de una tormenta moderada o grave de citoquinas, fueron tratados con metilprednisolona a dosis de 250 a $1000 \mathrm{mg}$ cada 24 horas por tres a cinco días, tocilizumab $8 \mathrm{mg} / \mathrm{kg} /$ día, repitiendo su dosis al día siguiente si persistía la inflamación (dosis máxima de $800 \mathrm{mg}$ ) y anticoagulación con HBPM en dosis profilácticas o terapéuticas, según los factores de riesgo protrombóticos del paciente y sus niveles de dímero D. De ellos, solamente el $8 \%$ requirió oxigenoterapia de alto flujo durante el ingreso hospitalario, teniendo todos estos obesidad grado I en adelante, diabetes mal controlada y síndrome de apnea obstructiva del sueño no tratado previamente. Este grupo necesitó también oxigenoterapia domiciliaria con cánula binasal a $3 \mathrm{lpm}$ en el momento del egreso hospitalario, durante uno a tres meses. Ninguno necesitó soporte ventilatorio invasivo, ni hubo fallecidos.

Las complicaciones asociadas al tratamiento con bolos de metilprednisolona, tocilizumab, HBPM o remdesivir de forma precoz fueron pocas, todas ellas leves, como el desarrollo de hiperglucemia leve a moderada (180-320 mg/ dl), que fue resuelta con el uso de insulina de rescate. No hubo casos de infecciones nosocomiales sobreañadidas con la profilaxis antibiótica instaurada con la inmunosupresión, ni casos de insuficiencia renal o citopenias asociadas al uso de remdesivir.

De la cohorte total, tres casos fallecieron $(0,34 \%$ de los casos); el primero, diabético sin tratamiento, quien consultó en su día 10 de sintomatología; el segundo un paciente diabético mal controlado con tratamiento hemodialítico por insuficiencia renal crónica, quien consultó en su día 8 de síntomas y el tercero diabético mal controlado, sin tratamiento farmacológico quien consultó en su día 8 de síntomas. Ninguno de los tres recibió corticoterapia en altas dosis, remdesivir o tocilizumab en ningún momento de su enfermedad, ya que fueron referidos a otros centros hospitalarios, debido a la alta carga asistencial en los hospitales pertenecientes a este estudio.

\section{Discusión}

La variabilidad y la gravedad de la enfermedad por coronavirus la determina la respuesta inflamatoria inmunológica hacia el virus. Dependiendo de la intensidad y el tipo de respuesta, así será el curso clínico y el pronóstico. En la mayoría de los pacientes, la respuesta inmunológica es adecuada y similar a la de cualquier resfriado común, pero en un porcentaje significativo, la respuesta puede ser exagerada, caracterizándose por una elevación intensa de citoquinas, entre ellas la interleucina 6, sustancias trombogénicas y altamente inflamatorias, que pueden llevar al paciente al daño de múltiples sistemas orgánicos, entre los más importantes el SDRA, miocardiopatía, polineuropatía, encefalitis, dermatosis, hepatitis o fracaso renal agudo, entre otros (10).

Existen factores de riesgo medibles, clínicos y analíticos, que son útiles para predecir si el paciente desarrollará dicha respuesta exagerada hacia el virus $y$, por tanto, se puede actuar de forma precoz para detener dicha respuesta, en un momento en el que el daño orgánico aún no esté instaurado.

Por esto proponemos el seguimiento estrecho de todos los pacientes infectados por COVID-19, enfatizando en un diagnóstico rápido y precoz de la infección, así como un análisis detallado de los 
factores de riesgo principalmente involucrados en una respuesta inmunológica fatídica, potencialmente tratable, si es detectada precozmente. Sugerimos realizar un estudio completo de los antecedentes de los pacientes y sus factores de riesgo conocidos, así como un análisis de posibles enfermedades mal tratadas o desconocidas hasta el momento de la infección por COVID-19, y su tratamiento oportuno. Recomendamos enfatizar en los seis factores de riesgo más frecuentes para una mala progresión de la enfermedad por COVID-19 y para el desarrollo de una tormenta de citoquinas, siendo estos, en orden decreciente de importancia: obesidad, diabetes mellitus mal controlada, síndrome de apnea obstructiva del sueño mal controlado, sexo masculino, edad mayor de 60 años e hipertensión arterial.

Recomendamos iniciar el remdesivir de forma precoz, desde el momento del diagnóstico, en pacientes con uno o más de los factores de riesgo mencionados, principalmente los que tienen obesidad, diabetes mellitus o síndrome de apnea obstructiva del sueño. La dosis recomendada es de $200 \mathrm{mg}$ al día el primer día, luego $100 \mathrm{mg}$ al día por 5 a 10 días, según la respuesta clínica y analítica.

Además, recomendamos un seguimiento estrecho de factores clínicos y analíticos, capaces de predecir el desarrollo incipiente de una tormenta de citoquinas, enfatizando en los días cinco a siete de síntomas, como la caída de la SpO2 <95\%, elevación de la FC $>95 \mathrm{lpm}$, fiebre $>38^{\circ} \mathrm{C}$, aparición de opacidades incipientes en vidrio deslustrado en la radiografía de tórax o alteraciones en uno o varios de los factores analíticos siguientes: interleucina 6 , dímero $\mathrm{D}$, ferritina, DHL, leuco o linfopenia, PCR, velocidad de sedimentación, marcadores de daño renal, hepático o cardíaco y tratar de forma enérgica y precoz, si se detectan las alteraciones predictoras del desarrollo de dicha tormenta $(10,11,12)$.

Si las alteraciones en dichos parámetros son leves, recomendamos el tratamiento rápido con metilprednisolona a dosis de $1 \mathrm{mg} / \mathrm{kg} /$ día y anticoagulantes en dosis profilácticas; si son moderadas o graves, recomendamos iniciar tratamiento con metilprednisolona en bolos de 250 a $1.000 \mathrm{mg}$ cada 24 horas por 3 a 5 dosis, tocilizumab a dosis de $8 \mathrm{mg} / \mathrm{kg} /$ día (siempre y cuando haya elevación de la interleucina 6 como componente de la tormenta de citoquinas) y anticoagulantes, a dosis ajustadas a los niveles de elevación de los reactantes inflamatorios y protrombóticos, así como de los factores de riesgo de cada paciente $(13,14,15,16)$.

Recomendamos un diagnóstico precoz de diabetes mellitus no diagnosticada previamente, así como un seguimiento estrecho de la glucometría, de los iones enfatizando en el potasio y de marcadores bioquímicos de sobreinfección bacteriana como la procalcitonina. Mantener una buena profilaxis antibiótica durante el tiempo que se utilicen los bolos de corticoides y el tratamiento con el tocilizumab. Corregir la hiperglucemia con hipoglucemiantes orales o insulinoterapia, según la gravedad del paciente y su riesgo de acidosis láctica. Hacer reposición de potasio en caso de hipopotasemia secundaria a la insulinoterapia $(17,18)$.

Es coherente pensar que el uso de remdesivir de forma precoz, en los primeros días de la enfermedad, cuando la carga viral es mayor, es más efectivo que su uso a posteriori, ya cuando la carga viral ha disminuido.

Lo mismo se debería pensar del uso de tocilizumab o la metilprednisolona, que serán más útiles cuanto menos daño estructural orgánico se haya desarrollado. La propuesta terapéutica es usar dichos inmunosupresores cuando apenas detectamos el inicio de la tormenta de citoquinas, sin haberse desarrollado un distrés respiratorio aún, de forma enérgica y oportuna, en vez de esperar a que el SDRA se haya instalado ya en el paciente. El tratamiento de un SDRA es mucho más complicado y de mucho peor pronóstico que el uso precoz de bolos de corticoides y tocilizumab al inicio de la tormenta inflamatoria $(19,20)$.

Los pacientes con SDRA y daño orgánico instaurado tardan meses en recuperarse o pueden incluso desarrollar lesiones irreversibles. El momento para usar la inmunosupresión es antes de que se haya dado este daño. Es aquí cuando la inmunosupresión tiene una mayor capacidad para cambiar el rumbo de la enfermedad y evitar el desarrollo de SDRA, el uso de ventilación mecánica, la mortalidad o las secuelas a largo plazo en los órganos del paciente $(17,20)$.

$\mathrm{Si}$ se usan dosis apropiadas de glucocorticoides, con o sin tocilizumab, ajustados al grado de inflamación que se está describiendo en la analítica del paciente, se puede detener la progresión del daño pulmonar y orgánico, pues con analíticas seriadas, se puede predecir y detener enérgica y precozmente el "estallido" de la tormenta de citoquinas. Con ello se reduce la necesidad de ventilación mecánica, oxigenoterapia en alto flujo, días en UCI, secuelas a mediano y largo plazo y la mortalidad. 
Con el manejo habitual, con dosis bajas de dexametasona, y el uso de tocilizumab y remdesivir solamente en casos de SDRA instaurado moderado o grave, la mayoría de estudios reportan daño miocárdico agudo en el 7.2 a $17 \%$ e insuficiencia renal aguda en el 2.9 a $15 \%$ de los casos graves. La incidencia de SDRA leve reportada ronda el $15 \%$, SDRA moderado y grave el 8 al $30 \%$ de los casos. La mortalidad global ronda el $2 \%$ para casos leves, $8 \%$ para SDRA ligero, $24 \%$ para SDRA moderado y del 50 al $80 \%$ para SDRA severo $(16,17)$.

\section{Conclusiones}

Es más fácil detener un "incendio forestal" cuando notamos la primera "chispa", que ya cuando se ha quemado todo el "bosque".

De los 796 pacientes vistos, solamente falleció un $0.37 \%$ de los casos, los cuales coincidieron en los siguientes aspectos: edad $>60$ años, sexo masculino, diabetes mal controlada, consulta tardía después del día 8 de síntomas y ausencia de tratamiento glucocorticoideo, remdesivir, anticoagulante y tocilizumab oportunos.

Los pacientes restantes, que corresponden al $99.6 \%$ de los casos, tuvieron una mortalidad y complicaciones del $0 \%$, con el manejo diagnóstico terapéutico arriba mencionado, consistente en:

1. El diagnóstico rápido de la infección por COVID-19, con alto índice de sospecha ante cualquier sintomatología catarral o sugestiva.

2. Seguimiento estrecho de la SpO2, frecuencia cardiaca, presión arterial, +/-glucometría, diarios.

3. La realización de analíticas de seguimiento entre los días cinco a siete y radiografías de tórax, buscando de forma precoz, indicadores del desarrollo de una tormenta de citoquinas.

4. Tratamiento oportuno de los pacientes que desarrollen dicha tormenta de citoquinas, con dosis y combinaciones de los medicamentos ajustadas a la gravedad de la inflamación documentada en la analítica. Esto incluye glucocoticoides, anticoagulación, tocilizumab y remdesivir. Los glucocorticoides se usarán solamente en caso de desarrollo de tormenta de citoquinas, con dosis ajustadas al grado de inflamación y los factores de riesgo de cada paciente. El tocilizumab se usará solamente en casos de tormenta de citoquinas moderada o grave, con elevación de interleucina 6 y el remdesivir se usará desde el diagnóstico en pacientes con uno o más factores de riesgo de mala progresión de la enfermedad.

5. No esperar al desarrollo de SDRA para comenzar con los medicamentos previamente indicados.

Iniciar un tratamiento precoz y oportuno en pacientes que desarrollen una tormenta de citoquinas puede evitar la progresión del daño pulmonar y orgánico de los pacientes con COVID-19, reduciendo la necesidad de oxigenoterapia a corto y largo plazo, la estancia hospitalaria, ingreso en UCI, la necesidad de ventilación mecánica y la mortalidad.

\section{Conflicto de interés}

Ninguno de los autores tiene conflictos de interés. Aprobación de comité de ética

Aprobado por el comité de ética del Hospital San Vicente en la Ciudad de Guatemala, con número de acta CEHSV-015-2021.

\section{Disponibilidad de datos}

Todos los datos generados y analizados que avalan esta investigación están disponibles para el editor a través del autor de correspondencia.

\section{Contribución de los autores}

Todos los autores han contribuido con la concepción, diseño del trabajo, obtención, interpretación y análisis de los datos, así como en la redacción y revisión del documento.

\section{Referencias}

1. Toniati P, Piva S, et al. Tocilizumab como tratamiento de la neumonía grave por COVID-19 con síndrome hiperinflamatorio e insuficiencia respiratoria aguda: un estudio de un solo centro de 100 pacientes en Brescia, Italia. Autoinmun Rev. 2020;19(7):102568. doi: https://doi. org/10.1016/j.autrev.2020.102568

2. Singh A, Singh $R$ et al. Remdesivir en COVID-19: Una revisión crítica de los estudios farmacológicos, preclínicos y clínicos. Diabetes Metab Synd. 2020;14(4):641-648. doi: https:// doi.org/10.1016/j.dsx.2020.05.018

3. Cortegiani A, Ippolito $\mathrm{M}$, et al. Justificación y evidencia sobre el uso de tocilizumab en COVID-19: una revisión sistemática. Pulmonolog. 2021;27(1): 52-66. doi: https://doi. 
org/10.1016/j.pulmoe.2020.07.003

4. Vidal Vademecum Spain. Madrid: Vidal Vademecum Spain. Monografía mecanismo de acción Remdesivir; 2021 [citado el 9 de noviembre del 2021]. Disponible en https:// www.vademecum.es/principios-activosremdesivir-j05ab16

5. Sreekanth R, O et a. Abordar el COVID-19 utilizando Remdesiviry Favipiravir como opciones terapéuticas. Chembiochem. 2020;22(6):939-948. doi: https://doi.org/10.1002/cbic.202000595

6. Vidal Vademecum Spain. Madrid: Vidal Vademecum Spain. Monografía mecanismo de acción Metilprednisolona; 2021 [citado el 9 de noviembre del 2021]. Disponible en https://www.vademecum.es/principios-activosmetilprednisolona-h02ab04

7. Vidal Vademecum Spain, Madrid: Vidal Vademecum Spain. Monografía mecanismo de acción Enoxaparina; 2021 [citado el 9 de noviembre del 2021]. Disponible en https://www. vademecum.es/principios-activos-enoxaparinab01ab05

8. Vidal Vademecum Spain. Madrid: Vidal Vademecum Spain. Monografía mecanismo de acción Rivaroxaban; 2021 [citado el 9 de noviembre del 2021]. Disponible en https://www. vademecum.es/principios-activos-rivaroxabanb01af01

9. Testa S, Prandoni P, Paoletti O, et al. Incremento sorprendente de los niveles plasmáticos de anticoagulantes orales directos en pacientes con síndrome respiratorio COVID-19 severo tratados con agentes antivirales: la experiencia de Cremona. J Thromb Haemost. 2020;18(6):13201323. doi: https://doi.org/10.1111/jth.14871

10. Prado Jerónimo CM, et al. Methylprednisolone as Adjunctive Therapy for Patients Hospitalized with Coronavirus Disease 2019 (COVID-19; Metcovid): A Randomized, Double-blind, Phase IIb, Placebo-controlled Trial, Clin. Infect. Dis. 2020;72(9):373-381. doi: https://doi. org $/ 10.1093 / \mathrm{cid} / \mathrm{ciaa} 1177$

11. Nelson BC, Laracy J, et al. Clinical outcomes associated with methylprednisolone in mechanically ventilated patients with COVID-19, Clin. Infect. Dis. 2020;72(9):367-372. doi: https://doi.org/10.1093/cid/ciaa1163

12. Edalatifard M, Akhtari $M$, et al. Methylprednisolone pulse as a treatment for hospitalised severe COVID-19 patients: results from a randomised controlled clinical trial. Eur. Respir. J. 2021;56(6);2002808. doi: https://doi. org/10.1183/13993003.02808-2020

13. Papamanoli A, Yoo J, et al. High-dose methylprednisolone in nonintubated patients with severe COVID-19 pneumonia. Eur J Clin Invest. 2020;51(2): e13458. doi: e13458. https:// doi.org/10.1111/eci.13458

14. Fadel R, Morrison AR, et al. COVID-19 Management Task Force, Early Short-Course Corticosteroids in Hospitalized Patients with COVID-19. Eur J Clin Invest. 2020;71(16):2114 2120. doi: https://doi.org/10.1093/cid/ciaa601

15. The WHO Rapid Evidence Appraisal for COVID-19 Therapies (REACT) Working Group. Association between Administration of Systemic Corticosteroids and Mortality among Critically Ill Patients with COVID-19: A Meta-analysis. JAMA. 2020;324(13):1330-1341. doi: 10.1001/ jama.2020.17023

16. Zhu HM, Li Y, Li BY, Yang S, Peng D, Yang X, Sun XL, Zhang M. Effect of methylprednisolone in severe and critical COVID-19: Analysis of 102 cases. World J Clin Cases. 2020; 8(23):5952-5961. doi: $10.12998 /$ wjcc.v8.i23.5952

17. Li X, Ma X. Acute respiratory failure in COVID 19: is it "typical" ARDS? Crit Care. 2020;24(198). doi: 10.1186/s13054-020-02911-9

18. Luo P, Liu Y, Qiu L, Liu X, Liu D, Li J. Tocilizumab treatment in COVID-19: A single center experience. J Med Virol. 2020;92(7):814818. doi: https://doi.org/10.1002/jmv.25801

19. Mikulska M, Nicolini LA, Signori A, Di Biagio A, Sepulcri C, Russo C, et al. Tocilizumab and steroid treatment in patients with COVID-19 pneumonia. PLoS ONE. 2021;15(8): e0237831. doi: https:// doi.org/10.1371/journal.pone.0237831

20. Liu J, Zheng X, Huang Y, Shan H, \& Huang J. Successful use of methylprednisolone for treating severe COVID-19. J. Allergy Clin Immunol. 2020;146(2):325-327. doi: https:// doi.org/10.1016/j.jaci.2020.05.021 